\title{
THE THIRTY-FOURTH ANNUAL MEETING OF THE AMERICAN MATHEMATICAL SOCIETY
}

The thirty-fourth Annual Meeting of the American Mathematical Society was held at Nashville, Tenn., Wednesday and Thursday, December 28-29, 1927, in conjunction with the meetings of the American Association for the Advancement of Science and the Mathematical Association of America. The headquarters of the mathematical organizations was at Ward-Belmont College. The meeting of the Society opened on Wednesday morning with a general session at which Professor James Pierpont delivered an address entitled Mathematical rigor, past and present. This address has already appeared in the January-February issue of this Bulletin. This was followed by sectional sessions: in the morning (i) Algebra and (ii) Point Sets and Foundations, and in the afternoon (i) Geometry and (ii) Analysis. The Gibbs Lecture was delivered on Wednesday afternoon, after the sectional sessions. Thursday morning was devoted to a general scientific session, followed by the annual business meeting and the election of officers and members of the Council. On Thursday afternoon was held a joint session of the two mathematical organizations and Section A of the American Association for the Advancement of Science.

The joint dinner on Thursday evening in the dining room of Ward-Belmont College was attended by about one hundred seventy-six mathematicians and their friends. Professor Archibald Henderson was toastmaster. After some general remarks on the significance of this meeting, he called for speeches by President Snyder, Professor Slaught, Professor Jewell C. Hughes, Dr. C. F. Roos, Professors Winger, R. L. Moore and Coolidge.

At the business meeting on Thursday morning, a resolution was adopted to put on record the "deep appreciation of the cordial hospitality and efficient services of all those 
concerned in making this meeting of the Society an exceptional success."

Thirty-four states and Canada were represented among the members present at this first general meeting of the Society in the South. The total number registered was nearly two hundred; the attendance included the following one hundred forty-five members of the Society:

Akers, F. E. Allen, R. B. Allen, Anderton, R. C. Archibald, Ashton, Atchison, R. W. Babcock, Barnhart, W. S. Beckwith, William Betz, Blichfeldt, J. D. Bond, Bowen, Boyd, E. W. Brown, Browne, H. E. Buchanan, Burington, Cairns, Carmichael, I. S. Carroll, Coleman, J. T. Colpitts, Coolidge, A. H. Copeland, L. P. Copeland, Crathorne, Dale, H. T. Davis, Denton, Doak, Dostal, Dresden, W. W. Elliott, G. C. Evans, G. W. Evans, Finkel, L. R. Ford, W. B. Ford, Fort, C. A. Garabedian, Gehman, J. L. Gibson, Hardin, E. R. Hedrick, Archibald Henderson, Herr, Hess, Holder, Hosford, A. M. Howe, J. C. Hughes, Huntington, Hyde, Louis Ingold, Ingraham, Dunham Jackson, C. G. Jaeger, E. H. Jones, B. F. Kimball, Kuhn, E. P. Lane, Larew, Lasley, Latimer, Harry Levy, F. A. Lewis, Luck, Lytle, MacDuffee, Mackie, Maddox, Maizlish, William Marshall, T. E. Mason, May, Messick, Michal, D. C. Miller, G. A. Miller, Mirick, Miser, U. G. Mitchell, Molina, C. N. Moore, R. L. Moore, Richard Morris, Morton, Mossman, Neelley, Olds, Oppenheim, Ore, Osborn, Ott, Palmer, Pierpont, Rainich, Rasor, Rawles, Reinsch, C. N. Reynolds, R. G. D. Richardson, Rider, H. L. Rietz, Risley, H. A. Robinson, Robison, Roos, Safford, Sanders, Sarratt, Saunders, J. H. Scarborough, Shaub, Sheffer, Shirk, Simmons, T. M. Simpson, Slaught, Smail, C. E. Smith, D. M. Smith, I. W. Smith, Virgil Snyder, R. P. Stephens, Stetson, Stouffer, Tappan, Waddell, L. E. Ward, Warren Weaver, W. P. Webber, G. T. Whyburn, Wiener, Wiley, K. P. Williams, A. H. Wilson, Winger, Frederick Wood, F. L. Wren, Wunder, J. M. Young, Yowell.

At the meeting of the Council, the following organization was elected to sustaining membership in the Society: The Penn Mutual Life Insurance Company, Philadelphia.

The following thirty-five persons were elected to ordinary membership:

Dr. Pedro Manuel Arcaya, minister of interior relations, Venezuela;

Miss Alma Bridgers Bizzell, Louisburg College;

Professor Edward Livingston Carr, Union University;

Dr. William Fitch Cheney, Jr., Tufts College;

Mr. John Alston Clark, Princeton University;

Lieut. George T. Derby, Corps of Engineers, United States Army;

Mr. Joe Lee Dorroh, University of Texas;

Mr. Nat Edmonson, Jr., Rice Institute;

Mr. Joseph M. Feld, Columbia University; 
Mr. Alfred Leon Foster, University of Valparaiso;

Mr. Francis Chauncey Hall, Rutgers University;

Professor Lena James Hawks, Ward-Belmont College;

Miss Deborah May Hickey, Rice Institute;

Miss Fannie Hopkins, University of Wisconsin;

Professor Charles Angevine Hutchinson, University of Colorado;

Mr. Harold John Kersten, University of Cincinnati;

Mrs. Mark Kormes, Hunter College;

Miss Ona Kraft, East High School, Cleveland;

Mr. Lloyd Lincoln Lowenstein, Cornell University;

Mr. Francisco Aniceto Lugo, electrical engineer, Maracaibo, Venezuela;

Mr. Edward Roy Cecil Miles, Rice Institute;

Miss Ethel Isabell Moody, Wells College;

Dr. Auguste Adolphe Nouel, Caracas, Venezuela;

Mr. Alexander Oppenheim, University of Chicago;

Mr. Henry Howes Pixley, Rutgers University;

Professor Luis José Fernandes Ribeiro, Pernambuco, Brazil;

Mr. Howard Lester Schug, Cornell University;

Dr. Stefan Serghiesco, Columbia University;

Mr. Leslie Donald Shriver, High School, Charleroi, Pa.;

Professor Charles Eugene Shull, Bridgewater College;

Mr. Ivan S. Sokolnikoff, University of Wisconsin;

Mr. Charles Chapman Torrance, Cornell University;

Mr. Franklin Grandy Williams, Cornell University;

Professor Rose Belle Wood, Greenville Womans College;

Dr. Oscar Zariski, Johns Hopkins University.

The following were elected to membership as nominees of Allyn and Bacon:

Miss Mary Virginia Kenny, Hunter College;

Mr. John Henderson Roberts, University of Texas;

as nominees of the National Life Insurance Company:

Mr. James Mayberry Earl, University of Minnesota;

Mr. Eli Gourin, Columbia University;

Mr. Neal Henry McCoy, University of Iowa;

Mr. Walter Lee Porter, Rice Institute.

The ordinary membership in the Society is now 1758 , including 166 nominees of sustaining members and 81 life members. There are also 36 sustaining members. The total attendance of members at all meetings during the past year was 690 ; the number of papers read was 404 . The number of members attending at least one meeting was 452 . At the annual election 252 votes were cast. 
The reports of the Treasurer and of the auditors ( $\mathrm{Mr}$. S. A. Joffe and Professor H. W. Reddick) showed a balance of $\$ 3608.67$, exclusive of the balances in the Bulletin, Transactions, Colloquium and special funds, and of the life membership reserve. The Society's Endowment Fund now has securities of par value $\$ 67,000$, yielding an annual income of $\$ 3125$; sustaining membership fees for the year amounted to $\$ 4600$. The amount received from sales of the Society's publications was $\$ 4831.57$.

The Board of Trustees did not meet at Nashville, lacking a quorum. At the adjourned meeting held in New York City, January 2, 1928, they adopted a budget showing estimated expenditures and receipts as $\$ 29,839.95$ and $\$ 28,846.66$ respectively.

The Librarian reported that the Library of the Society now contains 7548 volumes.

At the annual election, which closed on Thursday morning, the following officers and other members of the Council were chosen:

Vice-Presidents, Professors H. L. Rietz and J. W. Young. Secretary, Professor R. G. D. Richardson.

Associate Secretaries, Professors Arnold Dresden (one year) and M. H. Ingraham (two years).

Treasurer, Professor W. B. Fite.

Members of the Editorial Committee of the Bulletin, Professors D. R. Curtiss (two years) and E. R. Hedrick (three years).

Member of the Editorial Committee of the Transactions, Professor H. H. Mitchell.

Members of the Board of Editors of the American Journal of Mathematics, Professors E. W. Chittenden (five years), A. B. Coble (four years), and G. C. Evans (three years). Members of the Council, Professors W. B. Carver, W. C. Graustein, Louis Ingold, F. D. Murnaghan, and W. L. G. Williams.

The tellers appointed by President Snyder to count the ballots were Professor A. B. Morton and Dr. R. G. Archibald. 
The following appointments were reported: to represent the Society at the inauguration of President Burgstahler, of Cornell College, on Friday, November 18, 1927, Professor H. L. Rietz; to represent the Society at the American Mining Congress held in Washington, December 1-3, 1927, Dr. W. D. Lambert; as Committee on Arrangements for the Summer Meeting of 1928, Professors T. C. Esty (chairman), Arnold Dresden, W. A. Hurwitz, H. L. Rietz, A. H. Sprague, and J. W. Young.

At the meeting of the Council, Professor J. L. Coolidge was named to succeed Professor L. P. Eisenhart as one of the three representatives of the Society in the National Research Council. Professors Arnold Dresden and Tomlinson Fort were continued for 1928 as the representatives of the Society in the Council of the American Association for the Advancement of Science. As the committee on the award of the Bôcher Prize, which will take place at the Annual Meeting in 1928, the following were appointed: Professors E. T. Bell, R. D. Carmichael, and Solomon Lefschetz.

The fifth Josiah Willard Gibbs Lecture, entitled Resonance in the solar system, was delivered on Wednesday afternoon before an audience of 280 persons, by Professor E. W. Brown, of Yale University. It will appear in full in the May-June issue of this Bulletin. Professors M. B. Porter, Tomlinson Fort, and Solomon Lefschetz were appointed a committee to recommend to the Council a lecturer for 1928 .

At the joint session of the Society, the Mathematical Association, and Section A, held on Thursday afternoon, the following papers were read:

I. The notion of probable error in elementary statistics, by Professor E. V. Huntington, retiring Vice-President of Section A.

II. The human significance of mathematics, by Professor Dunham Jackson, retiring President of the Mathematical Association.

III. Some philosophic aspects of mathematics, by Professor Arnold Dresden. (Address delivered at the request of the 
American Mathematical Society.) This address will appear in full in an early issue of this Bulletin.

Titles and abstracts of the papers read at the regular sessions of the Society follow below. On Wednesday morning, President Snyder presided over the general session and the Section of Algebra, and Vice-President C. N. Moore over the Section of Point Sets and Foundations. On Wednesday afternoon, President Snyder presided over the Section of Geometry and during the Gibbs Lecture; Professor H. L. Rietz presided over the Section of Analysis. President Snyder also presided at the general session on Thursday morning. At the joint session on Thursday afternoon, Professor Dunham Jackson, chairman of Section A, presided, relieved during the presentation of his own address by Professor W. B. Ford, president of the Mathematical Association. The papers numbered 1-9 were read in the Section of Algebra, Nos. 10-20 in the Section of Point Sets and Foundations, Nos. 21-37 in the Section of Geometry, Nos. 38-53 in the Section of Analysis, and Nos. 54-70 in the general session. The papers of Frank Ayres, W. L. Ayres, Blumenthal, Browne (second paper), Camp, Campbell, Coble, Curtiss, Foster, Franklin, Hazlett, Hollcroft, Kasner, Mathews, Oppenheim, Ranum, Roberts, Roth, Sheffer (third paper), Veblen, Walsh, G. T. Whyburn (third and fourth papers), W. M. Whyburn, and Wilder were read by title. Mr. Holt was introduced by Mr. Burington.

1. Professor D. R. Curtiss: A class of diophantine equations with bounded positive integral solutions.

The equations here considered have the form $F\left(1 / x_{1}, 1 / x_{2}, \cdots, 1 / x_{n}\right)=k$, where $F$ is a polynomial with positive integral coefficients and $k$ is a positive integer. Every such equation is shown to have only a finite number of positive integral solutions, or no such solution. The proof consists in establishing an upper bound for each $x$. This proof also applies to certain equations in which the right hand side involves the variables $x$. An interesting corollary is the proposition that among the equations $x^{n}-a_{1} x^{n-1}+a_{2} x^{n-2}$ $-\cdots+(-1)^{n} a_{n}=0$ all of whose roots are positive integers, there are only a finite number (if any) whose coefficients satisfy a relation $F\left(a_{1} / a_{n}\right.$, $\left.a_{2} / a_{n}, \cdots, a_{n-1} / a_{n}\right)=k$, where $F$ and $k$ are as defined above. 


\section{Professor Olive C. Hazlett: Note on formal modular invariants.}

In a recent paper in Liouville's Journal, W. L. G. Williams proved a number of theorems to the effect that all polynomials of a certain type in the coefficients of a binary cubic $f$ (with binomial coefficients) are formal modular invariants of $f$ modulo $p$, where $p$ is a prime. The use of binomial coefficients makes his proofs not applicable when the order of the form is such that any of these binomial coefficients is congruent to zero modulo $p$. The present paper proves these theorems and a more general theorem which includes all of Williams' theorems as special cases for the general Galois field $G F\left[p^{n}\right]$ in which $p$ is any prime whatsoever and there is no restriction on the order of the binary form.

\section{Professor Olive C. Hazlett: Algebras A defined over an algebra $B$.}

This paper considers the theory of an algebra $A$ defined over all algebra $B$, where $B$ is an algebra defined over a field $F$. After proving that the only case which has any general significance is when $B$ is semi-simple, the paper proves that Wedderburn's fundamental theorem for a linear algebra over a field has a precise analogue here. Moreover, if an algebra $A^{\prime}$ over $F$ can be regarded as $A$ over $B$, then the canonical form of $A^{\prime}$ over $F$ is equivalent to the canonical form of $A$ over $B$; and thus the central results of Wedderburn's beautiful theory of a linear algebra over a field $F$ can be translated into the analogous results for a linear algebra over $B$.

4. Professor Oystein Ore: Arithmetical theory of Galois fields. First communication.

This paper contains the fundamentass of a new arithmetical theory of Galois fields which derives the ideal properties of the field directly from the defining equation, as in the author's theory of general algebraic fields (Mathematische Annalen, vols. 96-97). It is proved that in Galois fields the complete system of residue classes for powers of prime ideals can be obtained by successive adjunctions of roots of binomial and trinomial canonical congruences. By this method various new results are obtained; in particular it is shown that the Hilbert metacyclic groups for the prime ideals have a very special structure.

5. Mr. L. M. Blumenthal: On the definiteness of a quadratic form, with an application to the roots of an algebraic equation.

By mathematical induction and the use of a well known theorem of Sylvester, the author obtains the necessary and sufficient conditions that a real, algebraic, quadratic form be definite. By writing the ternary form $\Sigma_{0}{ }^{2} a_{i j} x_{i} x_{j}$ as a quadratic in $x_{0}$, it is shown to be definite if and only if its discriminant (a binary quadratic form in $x_{1}, x_{2}$ ) be positive for all real values of $\left(x_{1}, x_{2}\right)$ different from $(0,0)$. These conditions can be expressed in 
terms of the coefficients of the ternary form, and Sylvester's theorem is employed to obtain the standard form for them. A quadratic form whose discriminant is the same as that of a real algebraic equation is shown to be positive definite if and only if the roots of the equation are real and distinct. Applying the previously deduced conditions that a quadratic form be positive definite, the well known necessary and sufficient conditions for the reality of the roots of the equation are obtained.

6. Professor G. A. Miller: The number of systems of imprimitivity of transitive substitution groups.

The main theorem proved in this paper is as follows: The number of systems of imprimitivity of any transitive substitution group is equal to the number of proper subgroups of this group which separately involve as a proper subgroup a fixed one of the subgroups composed of all the substitutions of this transitive group which omit a given letter, and the number of letters in a set of such a system of imprimitivity is equal to the index of this fixed subgroup under the larger subgroup. By means of this theorem the author determines the number of systems of imprimitivity of any transitive group in which the degree of the subgroup composed of all the substitutions which omit a given letter is exactly half the degree of the group. In particular, if in a transitive substitution group $G$ the subgroup $G_{1}$ composed of all the substitutions of $G$ which omit a given letter is transitive and omits half the letters of $G$, then the number of systems of imprimitivity of $G$ is one more than the number of proper suhgroups of $G_{1}$.

7. Professor C. G. Latimer: On forms which repeat under multiplication.

In this paper it is shown that if $\left(A_{i j}\right)$ is the adjoint of the general Hermitian matrix $\left(a_{i j}\right),(i, j=1,2,3)$, then the Hermitian form $\bar{x}_{0} x_{0}+\sum_{i, j=1}^{3} A_{i j} \bar{x}_{i} x_{1}$ repeats under multiplication. This is seen to be a generalization of Hermite's self-reproductive quadratic form. From the above form we obtain an octenary quadratic form $\phi$, which also repeats under multiplication. By proper specialization of the parameters in $\phi$, we obtain E. T. Bell's recent generalization (Annals of Mathematics, (2), vol. 27, p. 99) of Degen's result on the sum of eight squares.

\section{Professor C. G. Latimer: On the primes in the general} cubic Galois field.

It is known that in every general cubic Galois field a rational prime, not a divisor of the discriminant, is either a prime of the field or is the product of three distinct prime ideals. It is found that a sufficient condition for a prime to be such a product is that it be a cubic residue of the rational integer $D$, where $D^{2}$ is the discriminant of the field $F$. If $F$ is of class unity, this condition is also necessary. It is shown that a necessary condition that $F$ be of class unity is that $D=9$ or $D=p$, a prime. F. S. Nowlan (this Bulletin, vol. 32 , pp. 374-80) has recently treated a problem equivalent to 
that of determining the primes in two special fields. Our results check with his.

\section{Dr. C. G. Jaeger: A character symbol for primes relative} to a cubic field.

A symbol $\psi(\pi)$ is defined with regard to the primes of a general cubic field analogous to the symbol defined by Dedekind in the study of the class number of a pure cubic field. It is hoped that this symbol will facilitate the study of the zeta function in the general cubic field.

10. Professor H. M. Gehman: Concerning irreducible continua.

If a bounded continuum $M$ is an irreducible continuum about a set $A$, then (1) $A^{\prime}$ (=A plus its limit points) contains some non-cut points of $M$; (2) $M$ is an irreducible continuum about those points of $A^{\prime}$ which are noncut points of $M$; (3) $A^{\prime}$ contains all points of $M$ which are such that there exists a neighborhood about the point of diameter less than any preassigned positive quantity whose removal leaves $M$ connected. As a corollary to (1) follows the well known theorem that every bounded continuum contains at least two non-cut points. The points mentioned in (3) are a special type of non-cut points.

11. Professor H. M. Gehman: On extending a correspondence in the sense of Antoine.

A continuous (1-1) correspondence between two plane points sets $M$ and $M^{\prime}$ can be extended in the sense of Antoine (Journal de Mathématiques, (8), vol. 4 (1921), p. 221), or $A$-extended, to their planes provided there exists a continuous (1-1) correspondence of the planes such that $M$ corresponds to $M^{\prime}$ under this correspondence. In this paper, continuous curves are classified by types according to the number of simple closed curves, end points, and branch points that they contain, and it is found that there are in all eighteen types for which a correspondence between two curves of the same type (and interior-class) can be $A$-extended. In particular, if $M$ is an acyclic continuous curve, a correspondence between $M$ and $M^{\prime}$ can be $A$-extended to their planes, if and only if either $M$ contains less than three branch points and the number of end points is finite, or $M$ has three branch points and five end points. Two other theorems are proved which generalize theorems of Antoine on the A-extension of a correspondence between sets consisting of collections of continuous curves.

12. Professor R. L. Moore: Concerning triods in the plane and the junction points of plane continua.

A triod is defined as a continuum containing a point $O$ (its emanation point) and consisting of three continua each of which is irreducible between $O$ and some other point and no two of which have in common any point except $O$. The point $O$ of a continuum $M$ is called a junction point of $M$, 
if $M$ contains a triod having $O$ as its emanation point and there exists a domain $D$ containing $O$ and such that $O$ is a cut point of the greatest connected subset of $M \cdot D$ that contains $O$. A bounded continuum $M$ is called triodic or atriodic according as there does not exist a subcontinuum $K$ of $M$ containing three distinct points and a continuum which contains no one of these points but which contains a point of every connected subset of $K$ that contains two of them. It is shown that, in the plane, (1) no continuum has uncountably many junction points, (2) there does not exist an uncountable set of mutually exclusive bounded triodic continua, (3) if $G$ is an uncountable set of mutually exclusive bounded continuous curves, then all but a countable number of them are either simple continuous arcs or simple closed curves.

\section{Professor R. L. Moore: On the separation of the plane by continua.}

This paper will appear in full in an early issue of this Bulletin.

14. Professor R. L. Moore: Concerning the sum of a countable number of closed point sets.

J. R. Kline has recently shown that if $G$ is a countable set of mutually exclusive bounded continua, and, for every positive number $\epsilon$, all but a finite number of them are of diameter less than $\epsilon$, then their sum is not connected. In the present paper it is shown that this theorem remains true if "continua" is replaced by "closed point sets."

\section{Professor G. T. Whyburn: On the structure of plane closed point sets which are accessible from certain subsets of their complements.}

If, in a plane $S, R_{1}, R_{2}$, and $R_{3}$ are mutually exclusive connected point sets, then $S-\left(R_{1}+R_{2}+R_{3}\right)$ contains not more than two points each of which is accessible from each of these sets. If every point of the closed and bounded point set $K$ is accessible from each of two mutually exclusive connected subsets of $S-K$, then either $K$ is a simple closed curve or there exists an arc which contains $K$. Let $K$ be an irreducible cutting of a continuous curve $M$ between two points $A$ and $B$ of $M$, and $R_{a}$ and $R_{b}$ the components of $M-K$ containing $A$ and $B$ respectively; then (1) $K$ lies in some single cyclic element of $M$; (2) if every point of $K$ is accessible from each of the sets $R_{a}$ and $R_{b}$, either $K$ is a simple closed curve or there exists an $\operatorname{arc} t$ such that $t \cdot M \equiv K$, and the boundary of no complementary domain of $M$ contains more than two points of $K$; if, in addition, the boundaries of no two complementary domains of $M$ have a common point, $M$ contains an $\operatorname{arc} T$ which contains $K$. Other consequences of these theorems are given.

16. Professor G. T. Whyburn: On the disconnection of continua. 
Let $M$ be any plane continuum. (1) If every subcontinuum of $M$ contains an uncountable collection of mutually exclusive point sets $[X]$ each of which cuts $M$, then every subcontinuum of $M$ is a continuous curve. (2) If, in (1), the point sets $[X]$ are countable, then every subcontinuum of $M$ contains an uncountable collection of mutually exclusive pairs of of points each pair of which cuts $M$. (3) If every subcontinuum of $M$ contains an uncountabie collection of mutually exclusive pairs of points each pair of which cuts $M$, then $M$ is a Menger regular curve. (4) The subcontinuum $N$ of $M$ contains an uncountable collection of mutually exclusive pairs of points each pair of which cuts $M$ if and only if $N$ contains an uncountable set of points each of which is either a cut point of $M$ or accessible from at least two complementary domains of $M$. (5) There exists a Menger regular curve $M$ containing an arc $t$ which contains a countable set of points $H$ such that no subset of $t-H$ cuts $M$.

\section{Professor G. T. Whyburn: On irreducible cuttings of continua.}

Let $M$ be any continuous curve; then (1) a cutting $K$ of $M$ is irreducible (see this Bulletin, vol. 33, p. 388) if and only if $K$ is the $M$-boundary of each component of $M-K ;(2)$ a cutting $K$ of $M$ between two points $A$ and $B$ of $M$ is an irreducible cutting between these points if and only if $K$ is the $M$-boundary of each of the two components of $M-K$ containing $A$ and $B$ respectively; (3) every cutting of $M$ between any two closed subsets $A$ and $B$ of $M$ contains an irreducible cutting of $M$ between $A$ and $B$. If every point of the irreducible cutting $K$ of a plane continuous curve $M$ between two points $A$ and $B$ of $M$ is accessible from each of the components $R_{a}$ and $R_{b}$ of $M-K$ containing $A$ and $B$ respectively, then either $K$ contains two points whose sum cuts $M$ or $R_{a}+R_{b}=M-K$ and $K$ is an irreducible cutting of $M$. Every bounded decomposable continuum $M$ which is an irreducible cutting of the plane has the property that however it be decomposed into two continua $H$ and $K$ neither $\equiv M, H \cdot K$ is the sum of two mutually exclusive continua.

\section{Dr. W. L. Ayres (National Research Fellow): Con- tinuous curves which are cyclically connected.}

A continuous curve $M$ is cyclically connected if and only if one of the following conditions holds: (1) if $K_{1}$ and $K_{2}$ are mutually exclusive closed subsets of $M$ each containing more than one point, there exist two mutually exclusive arcs, each of which has a point of $K_{1}$ as one end point and a point of $K_{2}$ as the other end point and lies in $M-K_{1}-K_{2}$ except for these points; (2) if, in (1), $K_{2}$ contains just one point $P$, the two arcs exist as in (1) except that they have just $P$ in common instead of being mutually exclusive; (3) if $x, y$, and $z$ are distinct points of $M$, there exist arcs $x y z$, $x z y, y x z$ of $M$; (4) (a) the boundary of every complementary domain of $M$ is a simple closed curve or an open curve, (b) no two unbounded complementary domains have a boundary point in common; (5) if $M$ is unbounded, (a) every point of $M$ lies on an open curve of $M$, (b) the same as (b) of (4). 
(Conditions 4 and 5 are for two dimensions only.) A number of conditions are given that every point of an unbounded continuous curve $M$ lie on an open curve of $M$.

\section{Mr. J. H. Roberts: Concerning continua that contain no} domains.

Menger has shown that if $P$ is a point of a plane continuum $M$ which contains no domain, for every positive number $e$ there exists a simple closed curve enclosing $P$ and of diameter less than $e$ and such that the set of points common to $M$ and this curve is totally disconnected. In the present paper it is shown that for every such curve $M$ there exists a continuous one-to-one transformation of the plane into itself such that in the transformed plane there exists a rectangular coordinate system with the following properties: (a) if $l$ is any line whatever parallel to one of the coordinate axes, then the set of points common to $l$ and $M$ is either vacuous or totally disconnected; (b) if $M$ is a continuous curve, and if $R$ is the interior of any rectangle whose sides lie respectively on the lines $x=a$, $x=b, y=c$, and $y=d$, where $a, b, c$, and $d$ are rational numbers, then the set of all points of $M$ that lie in $R$ is the sum of a finite number of maximal connected subsets and so is the set of all points of $M$ that belong to $R^{\prime}$.

20. Professor R. L. Wilder: Concerning R. L. Moore's axioms $\Sigma_{1}$ for plane analysis situs.

In his paper On the foundations of plane analysis situs (Transactions of this Society, vol. 17 (1916), pp. 131-164), R. L. Moore proposed a system, $\Sigma_{1}$, of eight axioms for plane analysis situs. The present paper establishes that the system $\Sigma_{1}$ may be reduced to a set of seven axioms, by the elimination of axiom 6, which is shown to be a consequence of the other axioms. It is also found, incidentally, that the property stated in axiom 7 may be used to characterize simple closed curve and open curve.

21. Dr. C. F. Roos (National Research Fellow) and Mr. Alexander Oppenheim: A symmetric method of fitting lines and planes.

The method usually followed to fit a straight line $y=a x+b$ to observed points $\left(x_{i}, y_{i}\right), i=1, \cdots, m>2$, is that of choosing $a$ and $b$ so as to minimize $\Sigma\left(a x_{i}+b-y_{i}\right)^{2}$. If both $x_{i}$ and $y_{i}$ are subject to error, there is no reason to prefer the line so obtained to the line $x=a^{\prime} y+b^{\prime}$, different from the first unless the given points are collinear, found by minimizing $\Sigma\left(a^{\prime} y_{i}+b^{\prime}-x_{i}\right)^{2}$. Using the hypothesis that fitting a straight line is independent of the choice of axes, we find that if $a x+b y+c=0$ is the line of fit, the most general function of $x_{i}, y_{i}, a, b$, and $c$ invariant under homogeneous strain, rotation, and translation is some power of $a x_{i}+b y_{i}+c$. If $a x_{i}+b y_{i}+c$ is proportional to the distance from $\left(x_{i}, y_{i}\right)$ from the corresponding point of the line of fit, we show that $a \cos \alpha+b \sin \alpha=\phi$, where $\phi$ does not involve $a, b$, and $c$, and $\tan \alpha$ is the ratio of the error in $y_{i}$ to that in $x_{i}$, assumed inde- 
pendent of $i$. We obtain for the line of fit a simple equation well adapted to calculation. The method generalizes to the fitting of planes and hyperplanes, and also offers a method of attack for the fitting of higher plane and space curves.

22. Mr. R. S. Burington and Mr. H. K. Holt: A classification of plane cubic curves under euclidean transformations.

Newton showed that every cubic is included in one of four canonical forms which he subdivided by methods partly geometric and partly algebraic into 72 types, with some omissions which have been supplied by later writers. These forms are not canonical forms as we use the term. The transformations which leave one of them unaltered are sometimes more than finite in number. Using a system of invariants and covariants, the authors obtain eight true canonical forms which give all cubic curves with at most a finite number of repetitions.

\section{Professor E. T. Browne: Involutions that belong to a linear class.}

We consider two square matrices $A, B$ of order $n$ which possess the property $A^{2}=B^{2}=I$, where $I$ is the unit matrix. If in addition $A$ and $B$ are such that every linear combination of them of the form $C=\xi A+(1-\xi) B$ also possesses the property $C^{2}=I$, we shall say that $A$ and $B$ determine a linear class of involutions of which $C$ is a member. In this paper a study is made of the properties of pairs of involutions that determine a linear class. The number and distribution of the linearly independent common fixed points and planes of $A$ and $B$ are determined, and necessary and sufficient conditions, both algebraically and geometrically, are given that $A$ and $B$ may determine a linear class. Finally, for $n=3$ and $n=4$ a special study is made of linear classes of harmonic homologies in the plane and of pointplane reflections and line reflections in space. A number of interesting results are obtained.

24. Professor J. H. Neelley: A note on the rational plane quartic curve with cusps or undulations.

This paper derives and discusses certain invariants and covariants of the rational plane quartic curve with either cusps or undulations. Also certain degenerate forms are considered.

\section{Professor R. M. Winger: Trinomial curves and mono- mial groups.}

Maschke (American Journal of Mathematics, vol. 17) has considered monomial groups in the ternary domain, which are derived from two generators of special type; his work was extended by Skinner (ibid., vol. 25). Both authors exclude the interesting groups that leave invariant the trinomial curves $x^{n}+y^{n}+z^{n}=0$. These curves, for rational values of $n$, have received attention from numerous writers (Loria, Spezielle Algebraische 
und Transzendente Ebene Kurven, vol. I, pp. $328 \mathrm{ff}$.), and particular cases have been studied in connection with their groups, notably by Muth $(n=3)$, Berzolari $(n=-2)$, Dyck $(n=4)$, Snyder $(n=5)$, Tappan and Musselman $(n=6)$. In a forthcoming paper the author treats in detail the case of the equianharmonic cubic $(n=3)$. The present paper generalizes the more important results for arbitrary integral values of $n$. The group is analyzed and the complete system of invariants, comprising four forms connected by a syzygy, is obtained. The fundamental invariant curves are discussed; in particular, conjugate sets of points, special and general, are constructed. The curves are also studied in relation to a quadratic inversion. Generalization to $n$-space is indicated.

\section{Professor H. A. Simmons: Strong and weak inequalities} involving the ratio of two chords or arcs of a circle; chains of inequalities.

Let two chords $c_{1}, c_{2}, c_{1}<c_{2}$, of a semi-circle be drawn, and call their intercepted $\operatorname{arcs} a_{1}, a_{2}$ respectively. It is well known that $\left(c_{1} / c_{2}\right)>\left(a_{1} / a_{2}\right)$. In the present paper certain areas and volumes that are naturally associated with the chords $c_{1}, c_{2}$ and arcs $a_{1}, a_{2}$ are compared. Special notions of "strong" and "weak" inequalities are introduced early in the paper, and form a large part of the basis of the discussion; they are used in stating theorems and corollaries. Probably the most interesting result of the paper is a chain of trigonometric inequalities appearing in the last section, and developed from the notion of strong inequality.

\section{Professor Louis Ingold: Transformations of the co- efficients of linear connection.}

In an earlier paper (Proceedings of the National Academy, vol. 11, pp. 252-256), the author applied a transformation to the coefficients $\Gamma_{n \lambda}^{\alpha}$ of an affine geometry, or a geometry of paths. This transformation was associated with a non-singular matrix $a_{j}^{i}$. In order to define the corresponding transformation in an arbitrary coordinate system, it was found desirable to regard the quantities $a_{j}^{i}$ as the components of a mixed tensor. In the present paper the same transformation is applied to the more general nonsymmetric coefficients $H_{i \lambda}^{8}$ and the invariants of the transformation are studied. A series of invariants, given implicitly in the eariier paper, are here formulated explicitly. New invariant properties are studied; for example, it is shown that if, in the original space, there exists a covariant tensor $h_{\sigma_{1} \cdots \sigma_{k}}$ whose covariant derivatives vanish, then the same is true of the transformed space.

\section{Professor A. B. Coble: The invariants of an algebraic curve under Cremona transformation.}

On page 394 of her treatise on Cremona transformations, Miss Hudson remarks that "the outstanding problem of the whole theory, in any number of variables, is still to determine the set of properties which are invariant 
for Cremona transformations but not for the wider group of Riemann transformations. The present author gives the following version of the matter for a non-hyperelliptic plane algebraic curve $f$ of genus $p \geqq 4$ : the Riemann invariants of $f$ are the projective invariants of the normal curve $C^{2 p-2}$ in $S_{2 p-1}$, the map of $f$ by its canonical adjoints; the Cremona invariants of $f$ are the simultaneous projective invariants of the normal curve and a particular rational surface, $\Gamma$, which contains $C^{2 p-2}$ as a simple curve. For the excluded cases, hyperelliptic or of genus $p \leqq 3$, special statements must be made. As $\Gamma$ varies for fixed $C^{2 p-2}$, we obtain the various types of plane curves $f$, all birationally equivalent, but all distinct under Cremona transformations.

\section{Professor M. C. Foster: On the envelope of lines deter- mined by centers of geodesic curvature.}

This paper is concerned with the envelope relative to the moving tangent plane of the lines which join the centers of geodesic curvature of the curves of an orthogonal system upon a surface. The paper considers the conditions that the point on the envelope coincide with certain special points on the rays of the normal rectilinear congruence formed by these lines.

\section{Professor M. C. Foster: On the loci of centers of geodesic} curvature.

Corresponding to every point on a surface $S$ referred to any orthogonal system are two centers of geodesic curvature, $G_{1}$ and $G_{2}$, of the curves $u=$ const. and $v=$ const. The loci of $G_{1}$ and $G_{2}$ are in general two surfaces which are called the $G$-surfaces for the particular orthogonal system on $S$. This paper is concerned with these surfaces corresponding to various orthogonal systems.

\section{Mr. Frank Ayres: Plane $n$-ic curves generated by pairs} of involutions on a line.

H. S. White has shown (American Journal, vol. 48 (1926), that any plane cubic curve can be generated by means of a pair of cubic involutions on a line. In the present paper the following general theorem is proved: Any plane $n$-ic curve whose equation, $(a x)^{n}=0$, can be transformed, by means of a collineation of the form $x_{j}=\alpha_{j} y_{1}+\beta_{j} y_{2}+\gamma_{j} y_{3},(j=1,2,3), \Delta \neq 0$, into one in which $y_{1}$ and $y_{2}$ are separated, can be generated by means of a pair of $n$-ic involutions on a line. For such $n$-ics two points exist such that the first polar of either with respect to the $n$-ic degenerates into $n-1$ lines through the other. Taking the coordinates of these points as the $\alpha_{j}$ and $\beta_{j}$ in the collineation, and $\gamma_{j}$ arbitrary, the required transformation can be effected. Moreover, $(\alpha)$ and $(\beta)$ are corresponding points on and intersection points of the 1 st, $\cdots,(n-3) \mathrm{d}$ Hessian and corresponding Steinerian of the $n$-ic; the line $(\alpha)(\beta)$ has $(n-\kappa-2)$-point contact with the $\kappa$ th Hessian and $(\kappa-1)$-point contact with the $\kappa$ th Steinerian at $(\alpha)$ and 
$(\beta)$. For a non-degenerate $n$-ic, the conic polar of each of the $n-2$ further points of intersection of $(\alpha)(\beta)$ and the Hessian has the intersection of the linear polars of $(\alpha)$ and $(\beta)$ with respect to the $n$-ic as its double point.

32. Professor A. D. Campbell: The discriminant of an $m$-ary quadratic in the Galois fields of order $2^{n}$.

The prime modulus 2 in the Galois fields of order $2^{n}$ makes the ordinary discussion of the discriminant $\Delta$ of the $m$-ary quadratic break down in these fields. In this paper it is shown that, for $m$ even, $\Delta$ is the determinant $D$ whose vanishing causes the first polar of every point $P$ with respect to the quadratic to pass through a fixed point $P^{\prime}$, where the modulus 2 is replaced by zero in $D$. On the other hand, for $m$ odd, every first polar passes through a fixed point $P^{\prime}$; in this case $D$ has 2 as a common factor, and $\Delta$ proves to be $D$ with the factor 2 removed and with 2 replaced by zero in the rest of $D$.

33. Professor A. D. Campbell: A note on the polar curves of plane algebraic curves in the Galois fields.

This paper appears in full in the present issue of this Bulletin.

34. Professor T. R. Hollcroft: On multiple points and curves of surfaces.

Five problems are solved in this paper, and the following characteristics of algebraic surfaces determined: (1) the maximum number of multiple points of given order on a surface of given order; (2) the maximum order of multiple points of which the surface of given order has a given number; (3) the maximum number of consecutive multiple points of given order on a surface of given order; (4) the maximum multiplicity of a curve of given order on a surface of given order with given multiple curves; (5) the maximum order of a curve of given multiplicity on a surface of given order with given multiple curves.

\section{Professor R. M. Mathews: Dual syzygetic pencils of cubics.}

The nine harmonic polars of an order cubic are dual to the nine flexes and form the base of a syzygetic pencil of class cubics dual to the pencil of order curves on the flexes. Properties of the curves of one pencil can be at once dualized for the other pencil. The present investigation is of figures whose points and lines are the same for both pencils. Among the theorems obtained is the following: Every order cubic of a syzygetic pencil cuts each class cubic of the dual pencil in a $G_{18}$ of points such that the tangents (line elements) at the points form an isomorphic $G_{18}$ on the class curve. The $G_{18}$ is that subgroup of Hesse's $G_{216}$ that leaves each curve invariant.

36. Professor Arthur Ranum: On spherical, quasi-spherical curves. 
A curve $C$ lying on a sphere $S_{1}$ whose tangents touch another sphere $S_{2}$, and two curves closely associated with it, are here investigated. If $S_{1}$ is not tangent to $S_{2}, C$ consists, in general, of an infinite number of congruent arches connected by cusps, but the number of arches may be finite and $C$ be a closed curve. If $S_{1}$ is tangent to $S_{2}$, the point of contact being $K, C$ consists of a single arch and $K$ is an asymptotic point of the curve. The equations of $C$ in general involve elliptic functions.

37. Professor Arthur Ranum: Spheres osculating a curve and quasi-osculating another curve.

An intensive study of the "quasi-osculating sphere" of a twisted curve, discovered in 1909 by Hostinsky and the author, leads in this paper to a variety of new curves, and also to a new principle of classification of curves. For instance, if $s, 1 / \rho$, and $1 / \tau$ are the arc length, curvature, and torsion, respectively, and if $\eta=\int d s / \tau$, then a curve that is osculated by the quasiosculating spheres of another curve is characterized by the intrinsic equation $\rho=a \sec \eta$, and since the equation $\rho=a \cos \eta$ is known to characterize a spherical curve, a close connection is established between the two classes of curves.

38. Professor L. R. Ford: On the limit points of discontinuous groups.

Certain theorems concerning the set of limit points of a properly discontinuous group of linear transformations are derived, the proofs being based on the properties of the isometric circle. The results are used to prove that a non-elementary group of non-loxodromic transformations is Fuchsian.

39. Professor G. M. Robison: On regularity of a certain class of definitions of summability of infinite products.

Let $P_{n}=\Pi_{k=1}^{n}\left(1+a_{k}\right)$ be the sequence corresponding to the infinite pro duct $\Pi_{n=1}^{\infty}\left(1+a_{n}\right)$. A new sequence $P_{n}{ }^{\prime}$ is defined linearly in terms of a set of constants $\left(\alpha_{n, k}\right)$ and the elements $\left(a_{k}\right)$ as follows: $P_{n}{ }^{\prime}=\Pi_{k=1}^{n}\left(1+\alpha_{n, k} a_{k}\right)$. If $\lim _{n \rightarrow \infty} P_{n}^{\prime}$ exists and is equal to $s$, the infinite product $\Pi_{k=1}^{\infty}\left(1+a_{k}\right)$ is said to be summable by the definition to the value $s$. This paper gives a criterion for the regularity of this type of definition. An example of a definition assigning a value to a divergent infinite product is also given.

40. Professor E. L. Mackie: The Jacobi condition for a problem of Mayer with variable end points.

The isoperimetric problem of minimizing the quotient of two simple integrals while keeping a third constant is considered, the three integrands being arbitrary functions of $x, y$, and $y^{\prime}$. The Euler-Lagrange differential equations are deduced as a first necessary condition on the minimizing arc, and a transversality condition is found, by application of the recent results of Bliss in his study of the problem of Mayer. A further necessary condition is then established for this problem by showing that an envelope 
theorem and a condition analogous to that of Jacobi apply to it as well as to simpler problems in the calculus of variations.

41. Professor P. R. Rider: Minimizing two types of definite integrals.

This paper considers the integrals $\int_{x_{1}}^{x_{2}} y^{n}\left(1+y^{\prime 2}\right)^{m} d x, \int_{x_{1}}^{x_{2}} y^{\prime n}\left(1+y^{2}\right)^{m} d x$. The equations of the extremals are derived, and also necessary and sufficient conditions for a minimum or a maximum. The results obtained for the second integral are used to extend the conclusions of two earlier papers by the author (this Bulletin, vol. 27, pp. 279-284; Annals of Mathematics, (2), vol. 24, pp. 167-174). The present paper will appear in the Annals of Mathematics.

42. Mr. T. H. Rawles: The invariant integral and the inverse problem in the calculus of variations.

In this paper a theory for the inverse problem in the calculus of variations is presented. The Hilbert invariant integral serves as a point of departure. By means of this integral we obtain the general form of the integrand function which is compatible with a prescribed relation between the slopes of the extremal and the normal to the transversal. Conditions are then found which determine when it is possible for a given family of curves to be the extremals of a problem involving this prescribed relation. Finally the particular form of integrand function is developed. The problem is discussed for $n$ variables in the ordinary and in the parametric form.

43. Dr. I. M. Sheffer (National Research Fellow): Systems of linear differential equations of infinite order with constant coefficients.

The linear differential equation of infinite order with constant coefficients, $\Sigma_{0}^{\infty} a_{n} y^{(n)}(x)=f(x)$, was solved by Schürer (Leipziger Berichte, vol: 70 (1918), pp. 185-240) for a certain class of analytic functions $f(x)$ and a certain class of constants $a_{n}$. The problem of solving $n$ such equations in $n$ unknown functions appears not to have been treated heretofore. It is the purpose of the present paper to effect this generalization. The method used is operational, and permits us to give a complete treatment for the class of functions considered.

44. Dr. I. M. Sheffer: An operational treatment of the linear differential equation of infinite order with polynomial coefficients of degree one.

The existence theorems for a linear differential equation of infinite order whose coefficients are polynomials of bounded degree have been considered by von Koch, Perron, Hilb, and others. We consider in this paper the case where the polynomials are of degree not exceeding one. The treatment of such an equation falls into two cases, according as a function $D(t)$, which introduces itself into the problem in a very natural manner, is or is not 
analytic throughout a certain region. In the former case, the problem can be reduced to the case of an equation with constant coefficients, treated in the preceding paper. If $D(t)$ is not analytic everywhere in the region considered, the discussion is more elaborate. In some cases solutions can be obtained in expansions of certain related functions. In every case, however, it is shown how the original equation can be reduced to a linear differential equation of finite order, of Fuchsian type. The treatment is largely operational.

45. Dr. I. M. Sheffer: On a region of analyticity of a class of analytic functions.

Let $F(x)=\Sigma_{0}^{\infty} F_{n} x^{n}$ be analytic about the origin, and let $f(x)=\Sigma_{0}^{\infty} F_{n} x^{n} / n$ ! be the associated entire function. If we are given $f(x)$ with the single property that superior limit $\left(\left|f^{(n)}(0)\right|\right)^{1 / n}=\rho$, the most that can be said of $F(x)$ is that it has $|x|=1 / \rho$ as its circle of convergence. It is reasonable to expect that if we know more about $f(x)$ we can determine a larger region of analyticity for $F(x)$. The writer has had occasion to consider the following class of functions $f(x): f(x)=e^{-\xi x} g(x)$, where superior limit $\left(\left|g^{(n)}(0)\right|\right)^{1 / n}=\lambda$, and $\xi$ is any constant (real or complex). For $\lambda \geqq|\xi|$ and for $\lambda<|\xi|$ we obtain regions of analyticity for $F(x)$ that are much more extended than the circle of convergence mentioned above. Borel summability is used to establish these results.

46. Professor C. C. Camp: Devices for computing the rates together with a statistical study of building and loan associations.

The author compares methods of computing the rates for building and loan associations in order to secure a maximum of accuracy with a minimum of numerical work. Among the methods examined are the following: interpolation with first, and with second differences; interpolation in sevenfigure logarithms from Glover's tables; rule of double false position using logarithms; simple iteration; iteration accelerated by the author's method; Newton's method; and that of binomial expansions. A good random sample of 28 out of 852 associations in Illinois shows a poor correlation between the borrower's and the investor's rates. If we omit the five highest rates for loans, a coefficient of $r=0.775 \pm 0.056$ is found, and the line of best fit, $y-0.0815=0.69(x-0.0688)$, is obtained. Without this omission the borrower's rate averages $8.87 \%$ as against $6.87 \%$ for the investor.

47. Professor Philip Franklin: Sets of functions, orthogonal. on an infinite interval, having the power of the continuum.

The theory of almost periodic functions treats of the expansion of such functions in terms of the sines and cosines which form a non-enumerable set of functions, orthogonal on an infinite interval. We here extend some of the properties of functions orthogonal on a finite interval to general sets of functions of this type. With regard to the existence of sets which are not linear combinations of a finite number of sines and cosines, we show that a complete set of such functions can be constructed from any (enumerable) complete set on a finite interval. 
48. Professor Philip Franklin: Approximation theorems for generalized almost periodic functions.

The almost periodic functions of $\mathrm{H}$. Bohr are originally defined as continuous functions, having displacement numbers $t(\epsilon)$, such that $D(t)=|f(x+t)-f(x)| \leqq \epsilon$, distributed in a regular fashion. The approximation theorem for these functions identifies them with the class of functions uniformly approximable by trigonometric polynomials. Several generalizations of the notion have been given by Stepanoff, Wiener, Besicovitch, and Weyl, each definition requiring less than continuity, and correspondingly weakening the sense in which $D(t)$ must be small for a displacement number. In this paper we show that for all these generalized almost periodic functions, the approximation theorem has an analogue, the functions of each type being completely equivalent to a class of functions approximable in some sense by trigonometric polynomials.

49. Professor Philip Franklin: A set of continuous orthogonal functions.

A set of orthogonal functions was constructed by Haar (Mathematische Annalen, vol. 69 (1910)) which had the property that the development of any continuous function in terms of these functions converged. The functions themselves were not continuous. In the present paper we construct a set of continuous functions which, we show, possesses the same property as Haar's. Our functions are obtained by normalization from broken-line functions.

50. Professor Philip Franklin: Almost periodic recurrent motions.

Recurrent motions, a special type of limiting motion associated with the stable trajectories of a steady dynamical system, have been defined and discussed by Birkhoff (Bulletin, Société Mathématique de France, vol. 40 (1912)). If we define an almost periodic motion as one whose coordinates are almost periodic functions (in the sense. of $\mathrm{H}$. Bohr) of the time, we note at once that every almost periodic motion is recurrent. For systems satisfying a certain stability condition, we show that conversely every recurrent motion is almost periodic.

\section{Dr. W. M. Whyburn (National Research Fellow): An existence theorem for differential systems.}

In this paper it is shown that there exists a unique set of absolutely continuous functions $y_{1}(x), \cdots, y_{n}(x)$, on the interval $X: a \leqq x \leqq b$, such that if $x=c$ is any point of $x$ and $a_{1}, a_{2}, \cdots, a_{n}$ are given constants, then $y_{i}(c)=a_{i}, i=1,2, \cdots, n$, and such that this set of functions satisfies the following system of differential equations almost everywhere on $X$ : $d y_{i} / d x=\sum_{j=1}^{n} A_{i j}\left(x, y_{1}, \cdots, y_{n}\right) y_{j}+f_{i}\left(x, y_{1}, \cdots, y_{n}\right), i=1,2, \cdots, n$, where the $A_{i j}$ and $f_{i},(i, j=1, \cdots, n)$, are functions summable (Lebesgue) of $x$ on $X$ for each fixed $\left(y_{1}, \cdots, y_{n}\right)$, continuous functions of $\left(y_{1}, \cdots, y_{n}\right)$ for 
each fixed $x$ on $X$, and finally there exist functions $M(x)$ and $L(x)$ that are summable on $X$ such that for all $\left(y_{1}, \cdots, y_{n}\right),\left(z_{1}, \cdots, z_{n}\right)$, and every $x$ on $X,\left|A_{i j}\left(x, y_{1}, \cdots, y_{n}\right)\right| \leqq M(x),\left|f_{i}\left(x, \quad y_{1}, \cdots, \quad y_{n}\right)\right| \leqq M(x)$, and $\left|A_{i j}\left(x, y_{1}, \cdots, y_{n}\right)-A_{i j}\left(x, z_{1}, \cdots, z_{n}\right)\right| \leqq L(x) \sum_{k=1}^{n}\left|y_{k}-z_{k}\right|, \mid f_{i}\left(x, y_{1}, \cdots, y_{n}\right)$ $-f_{i}\left(x, z_{1}, \cdots, z_{n}\right)\left|\leqq L(x) \Sigma_{k=1}^{n}\right| y_{k}-z_{k} \mid,(i, j=1,2, \cdots, n)$. A method of successive approximations is used together with a lemma which shows that the approximating functions are uniformly bounded.

52. Dr. W. M. Whyburn (National Research Fellow): On the functional relation between the solutions of differential systems and the initial conditions.

The differential system considered in the author's paper $A n$ existence theorem for differential systems (see above) is discussed with the initial conditions $y_{i}\left(a_{i}\right)=b_{i}, i=1, \cdots, n$, where $b_{1}, \cdots, b_{n}$ are constants and $a_{1}, \cdots, a_{n}$ are points of the interval of definition, $X$. A positive constant $\delta$ is determined such that if all the points $a_{i}$ lie on an interval $D$ of length less than or equal to $\delta$, there exists a unique solution of the given differential system which satisfies the initial conditions. The above-mentioned existence theorem is used to extend this solution throughout $X$. It is shown that the solution is continuous in the $2 n+1$ variables $\left(a_{1}, \cdots, a_{n}, b_{1}, \cdots, b_{n}, x\right)$ throughout the manifold defined by $\left|b_{i}\right| \leqq B, a_{i}$ and $x$ on $D, i=1, \cdots, n$, where $B$ is a constant. Other properties of the solutions of this system are discussed.

53. Professor J. L. Walsh: On approximation to an arbitrary function of a complex variable by polynomials.

In the z-plane let two Jordan curves lie exterior to each other except for a single point which they have in common. Let the point set $M$ consist of these curves and their interiors. If the function $f(z)$ is continuous on $M$ and analytic in the interior points of $M$, then on $M$ this function can be uniformly expanded in a series of polynomials in $z$. If the bounded point set $M$ consists of a finite number of Jordan arcs and does not divide the plane, then an arbitrary function $f(z)$ continuous on $M$ is developable on $M$ in a uniformly convergent series of polynomials in $z$.

54. Professors D. J. Struik and Norbert Wiener: The Heisenberg theory of quanta under general five-dimensional relativity.

The present paper represents a reconciliation between general relativity and the Heisenberg idea that the Schroedinger wave equation determines a flow of probabilities. By the introduction of the Kaluza five-dimensional theory, the complex probabilities of Hilbert and Heisenberg are made real. The theory assumes a form analogous to that of sound under general relativity and at infinitely high temperatures. It offers the possibility of an interpretation of $m, e$, and $h$ as dimensional constants. The recent work of Schidlof, identifying as it does the proton and the electron, indicates that $M$ may also be explained as a dimensional constant. 
55. Professors C. A. Garabedian: On thick circular and rectangular plates loaded at the center.

In the forthcoming number of the Journal de l'Ecole Polytechnique will appear a paper (Correction de certains résultats sur la flexion d'une plaque circulaire épaisse donnés par de Saint-Venant....) in which the author shows the necessity of revising certain currently accepted solutions in thick plates under central load. The solutions in question are based upon an erroneous argument first advanced by de Saint-Venant. The cases of interest are those in which the plate is of circular or rectangular contour and clamped or supported at the edge, making in all four problems to be solved anew. The present paper obtains the solutions of these problems, and notes that the correct formulas, as distinguished from those hitherto published, give a consistent stress distribution at the point of application of the concentrated load.

\section{Professor G. Y. Rainich: Rotations in four-space.}

In the six-space the vectors of which represent infinitesimal rotations or antisymmetric tensors of a euclidean four-space, two absolutely perpendicular three-spaces are introduced which are invariant under all rotations in the four-space. Every six-vector is the sum of two three-vectors of opposite type, i.e., of two vectors of the three-spaces mentioned above. The known invariants and simultaneous invariants of six-vectors are simply expressed in terms of the three-vectors. A direction of a plane in fourspace can be given by two unit vectors of opposite type, which in turn are determined by the plane. Two simultaneous invariants of three six-vectors which appear to be novel are introduced as triple alternating products of three-vectors. In a special case these two simultaneous invariants characterize the relative position of three planes in four-space. They have been used by the author in his recent work on integrals of tensor fields. The decomposition of an infinitesimal rotation corresponds to the known decomposition of a general rotation in four-space into two elementary rotations.

57. Professor E. R. Hedrick: Stieltjes integrals in the complex plane.

The definitions and the properties of Stieltjes integrals in a complex plane are studied, and theorems analogous to those of the traditional theory of functions are stated.

58. Professor C. N. Moore: On certain criteria for the summability of integrals.

In this paper there is determined a set of necessary and sufficient conditions that a given infinite integral be Cesàro summable of order $n$. If the integral is $\int_{0}^{\infty} \gamma(x) d x$, the conditions involve the existence of a set of solutions of the equations $\gamma_{0}(x)=\gamma(x), \gamma_{s-1}(x)=-x \gamma_{s}{ }^{\prime}(x),(s=1,2, \cdots, n+1)$ such that $\int_{0}^{\infty} \gamma_{i}(x) d x$ is summable $(C, n-i),(i=1,2, \cdots, n+1)$. The re- 
sults are in the main analogous to some theorems regarding summable series due to Hardy and Littlewood (see Mathematische Zeitschrift, vol. 19 (1923), pp. 71-74), but there are also certain departures from this analogy, as is frequently the case in passing from theorems for divergent series to corresponding theorems for divergent integrals.

\section{Professor Dunham Jackson: On certain problems of} approximation in the complex domain.

A method involving Bernstein's theorem on the derivative of a trigonometric sum or a polynomial, or some form of generalization of it, has been found useful in the treatment of various problems in the approximate representation of real functions. The present paper illustrates the applicability of the method in proving the convergence of certain processes of approximation over a region of the complex plane.

60. Dr. C.F. Roos (National Research Fellow): On a type of Stieltjes integral equations.

In the study of linear functionals, especially in connection with Riesz's theorem, it is desirable to know something about the solutions of systems of Stieltjes integral equations of the type $u_{h}(x)=f_{h}(x)=\int_{a} v K_{h}(x, \mathrm{~s}) d u_{\theta}(s)$, $(h, g=1, \cdots, m)$, where $g$ is an umbral index. The present paper gives a method which does not make use of an integration by parts. The resolvent kernel is a function which in a certain general sense is reciprocal to $K_{h g}(x, s)$.

61. Professor J. W. Lasley: Note on the line equation of a plane curve.

The projective differential geometry of plane curves associates with every curve an ordinary linear homogeneous differential equation of the third order. The Lagrange adjoint of the latter is satisfied by the line coordinates of the curve. The parametric point equations furnish integrating factors for this adjoint. The integrations can be performed, and the general solution of the adjoint equation obtained. There is, however, much latitude in the choice both of dependent and independent variables, and this choice materially affects the differential equations involved, Moreover, one must be careful that the fundamental system of solutions finally obtained leads to the original curve and not merely to some projective transform of it. The present paper outlines a process for effecting this transition from the point to the line equations of the given plane curve.

62. Mr. W. E. Roth: Solution of the polynomial equation $P(A, X)=0$ in matrices. Preliminary communication.

The equation $\mathrm{P}(A, X) \equiv F_{0}(A) X^{p}+F_{1}(A)^{p-1}+\cdots+F_{p}(A)=0$, where the $F_{i}(A), i=0,1, \cdots, p$, are polynomials in the given matrix $A$, is solved in the present paper for such values of $X$ as are expressible as polynomials in $A$. A criterion for the existence of such solutions is established, and their explicit form is produced. 


\section{Professor Edward Kasner: Geometric characterization of the derivative congruence of a general polygenic function.}

In an earlier paper, read at the October meeting of the Society, the author showed that the derivative $r=d w / d z$ of a general polygenic (i.e., non-monogenic) function $w$ is completely represented by a congruence of clocks, a definite clock corresponding to each point $z$. (See Science, vol. 66 (1927), p. 581, and Proceedings of the National Academy.) If, however, we are given an arbitrary congruence whose clocks are in arbitrary correspondence with the points $z$, it will not in general be possible to identify this situation with the derivative of any function. Necessary and sufficient conditions are found, first in analytic, then in geometric form. If the congruence and correspondence are defined by a central vector $A$ and a phase vector $B$, where $A$ and $B$ are represented as complex (in general polygenic) functions, then $\left(D_{x}-i D_{y}\right) A=\left(D_{x}+i D_{y}\right) B$, that is $\mathfrak{P} A=\mathfrak{D} B$, in terms of the operators whose geometric meaning is given in the earlier paper. The present paper will be sent to the Comptes Rendus.

\section{Professor Edward Kasner: A new interpretation of the Laplacian operator.}

The Laplacian operator $\Delta$ is expressible in the form $\Delta=4 \mathfrak{D P}=4 \mathfrak{B D}$, where $\mathfrak{D}$ denotes the mean derivative or central operator, and $\mathfrak{B}$ the phase operator defined in the papers cited above. If $w$ is any complex function, we find that $\Delta w=4 \Gamma \mathfrak{D} \Gamma \mathcal{D} w$, where $\Gamma$ denotes the operation of taking the conjugate complex value. Hence the Laplacian of any function (real or imaginary) is four times the conjugate of the mean derivative of the conjugate of the mean derivative of that function. This paper will be offered to this Bulletin.

65. Professor Edward Kasner: Homographic and uniform clocks.

If a pencil of lines is put into one-to-one or homographic correspondence with the points of a circle, we call this a general or homographic clock. If the angle between two lines varies in proportion to the circular arc determined by the corresponding points, we call the clock uniform. (Necessarily the angular measure of the arc is then twice the angle between the lines.) Uniform clocks may be positive or negative according to whether the sense agrees with the sense of the pencil or disagrees. The clocks in the author's theory of polygenic functions thus appear in the present general theory as negative uniform clocks. For the general case, the centroid is distinct from the center.

66. Professor Oswald Veblen: Projective tensors and connections.

The classical theory of differential invariants may be based on the observation that any analytic transformation of coordinates determines a linear transformation in a unique way. It is also true that any analytic 
transformation determines a unique linear fractional transformation. The coefficients of this linear fractional transformation are the transformation coefficients of the components of a class of invariants, called projective tensors, which havealgebraic properties analogous to those of ordinary tensors and seem to provide the formalism needed for a group of problems to which the ordinary tensor analysis is not adequate. This paper contains an introductory account of the theory and will be published in the Proceedings of the National Academy of Sciences.

\section{Professor E. T. Browne: Note on the chacteristic equa- tion of a matrix.}

This paper will appear in full an early issue of this Bulletin.

\section{Professor E. P. Lane: The projective differential} geometry of systems of linear homogeneous differential equations of the first order.

The author considers first the projective geometry, in a linear space of $n$ dimensions, of a system of $n+1$ linear homogeneous differential equations of the first order in one independent and $n+1$ dependent variables. The transformation of dependent variables that is used is determined by the configuration to be studied, which in any case consists of a set of varieties each of which is generated by $\infty^{1}$ linear spaces, with the generators in correspondence. The precise number of possible configurations is determined. Certain curves called intersector curves on a variety are defined, and the locus of their tangents is investigated. In 5-dimensional space, the intersection of a point configuration with a hyperquadric yields a geometric interpretation in ordinary ruled space. A complete system of invariants is furnished for two ruled surfaces in ordinary space, and a new canonical form of the differential equations therefore is derived. The foundations are laid for the projective differential geometry of a set of varieties generated by $\infty^{k}$ linear spaces, with the generators in correspondence. The system of equations for such a configuration consists of $k(n+1)$ linear homogeneous partial differential equations of the first order in $n+1$ dependent and $k$ independent variables, the coefficients of which satisfy $\frac{1}{2} k(k-1)(n+1)^{2}$ conditions of complete integrability.

\section{Professor G. T. Whyburn: Concerning accessibility} in the plane.

In this paper the following theorems are proved. (1) If $G$ is a countable collection of mutually exclusive connected point sets in a plane $S, T$ denotes the sum of all the point sets of the collection $G$, and $K$ denotes the set of all points $X$ in $S-T$ such that $X$ is accessible from at least three of the sets of $G$, then $K$ is countable. (2) If every point of a plane continuum $M$ is accessible from at least two complementary domains of $M$, then $M$ is a Menger regular curve, and if $M$ is bounded, its cut points are countable. (3) In order that the boundary point $P$ of a connected open subset $R$ of 
a continuous curve (in any number of dimensions) should be regularly accessible from $R$, it is necessary and sufficient that $R+P$ should be connected im kleinen at $P$. As a corollary to (1) we have the result that the set of all points of a plane continuum $M$ each of which is accessible from at least three complementary domains of $M$ is countable.

70. Mr. Alexander Oppenheim: The approximate functional equation for the multiple theta function, and the associated trigonometric sums.

In this note the approximate functional equation for the multiple theta function is obtained by a process of induction from a slight extension of the corresponding equation for the simple theta function. The latter formula was first given by Hardy and Littlewood in 1914, and various proofs of it have since been published. Application is also made after the manner of Hardy and Littlewood to the study of certain trigonometric sums associated with the powers of a simple theta function.

ARnold DRESDEN, Associate Secretary

\section{A CORRECTION}

By O. D. KELLOGG

Dr. F. Vasilesco has kindly called my attention to an error in my symposium address, Recent progress with the Dirichlet problem, which appeared in this Bulletin (vol. 32 (1926), pp. 601-623).

On page 620 , in the second paragraph of $\S 8$, the property (b) should read "the part of $B$ in any closed region $T^{\prime}$ has capacity 0 "; and the last phrase in this same paragraph should read "then $B$ must have the property $(b)$."

The footnote at this point should refer to a paper by Dr. Vasilesco, Sur les singularités des fonctions harmoniques, which will appear in the volume of the Journal de Mathématiques for this year, which is to be dedicated to M. Picard.

HaRVARD UNIVERSITY 